\title{
THE NUMBER OF LATTICE POINTS IN AN $n$-DIMENSIONAL TETRAHEDRON ${ }^{1}$
}

\author{
S. CHOWLA AND W. E. MIENTKA
}

Let the $a_{i}(1 \leqq i \leqq n)$ be positive integers, no two of which have a common factor; let $A$ denote the product of the $a_{i}$.

In this paper we prove the following two exceedingly simple theorems, ${ }^{2}$ which we have not, to our surprise, seen before.

THEOREM 1. If $\eta \equiv 0(\bmod A)$, the number of solutions of

$$
\sum_{i=1}^{n} a_{i} x_{i} \leqq \eta, \quad x_{i} \geqq 0 \quad(1 \leqq i \leqq n)
$$

is a polynomial with rational coefficients in the quantities $\eta / A$ and the $a_{i}$.

THEOREM 2. If $\eta \equiv 0(\bmod A)$, the number of solutions of

$$
\sum_{i=1}^{n} a_{i} x_{i}=\eta, \quad \text { each } x_{i} \geqq 0,
$$

is a polynomial with rational coefficients in the quantities $\eta / A$ and the $a_{i}$.

One should mention here that both theorems are completely trivial for $n=1$. Theorem 2 is also trivial for $n=2$, and indeed for the following reason. Clearly if $\eta \equiv 0\left(\bmod a_{1} a_{2}\right)$,

$$
a_{1} x_{1}+a_{2} x_{2}=\eta
$$

implies that $x_{1} \equiv 0\left(\bmod a_{2}\right), x_{2} \equiv 0\left(\bmod a_{1}\right)$. Let $x_{1}=a_{2} x_{1}^{\prime}, x_{2}=a_{1} x_{2}^{\prime}$. Hence we see that if $\eta \equiv 0\left(\bmod a_{1} a_{2}\right)$ the number of solutions of

$$
a_{1} x_{1}+a_{2} x_{2}=\eta, \quad x_{1} \geqq 0, x_{2} \geqq 0
$$

is the same as the number of solutions of

$$
x_{1}+x_{2}^{\prime}=\frac{\eta}{a_{1} a_{2}}, \quad x_{1}^{\prime} \geqq 0, x_{2}^{\prime} \geqq 0
$$

which is clearly $\left(\eta / a_{1} a_{2}\right)+1$.

However, Theorem 2, while not deep, does not seem to be trivial for $n=3$.

Received by the editors March 2, 1955.

${ }^{1}$ This research was supported by the United States Air Force through the Office of Scientific Research of the Air Research and Development Command.

2 For the case when the $a_{i}$ 's are all equal to 1 , the result is well known. 
ExAmple. For $n=3$ (in Theorem 2) and $\eta \equiv 0\left(\bmod a_{1} a_{2} a_{3}\right)$, the number of solutions of $\sum_{i=1}^{3} a_{i} x_{i}=\eta$ is

$$
\frac{\eta^{2}}{2 a_{1} a_{2} a_{3}}+\frac{\eta\left(a_{1}+a_{2}+a_{3}\right)}{2 a_{1} a_{2} a_{3}}+1 .
$$

Theorems 1 and 2 are proved as follows. Following D. H. Lehmer [1] and D. C. Spencer [2] we write $L(\eta)$ for the number of solutions of (1), when $\eta$ is any non-negative real number; we use $r(\eta)$ to denote the number of solutions of (2). Observe that $r(\eta)=0$ if $\eta$ is not an integer. We also write

$$
L^{*}(\eta)=L(\eta)-r(\eta) / 2 .
$$

We have (see [2])

$$
L^{*}(\eta)=\frac{1}{2 \pi i} \int \frac{e^{\eta S} d S}{S \prod_{i=1}^{n}\left(1-e^{-a_{i} S}\right)}
$$

where the integral is along the vertical line $\sigma=c(c>0 ; S=\sigma+i t, \sigma$ and $t$ real). Now $L^{*}(\eta)$ is a polynomial in $\eta$ of degree $n$, where the coefficients $B_{i}(1 \leqq i \leqq n)$ of $\eta^{i}$, multiplied by $A$, are polynomials in $a_{i}$. The constant term $B_{0}$, which is a function of the $a_{i}$ and $\eta$, is periodic in $\eta$, with a period equal to $A$. (See G. H. Hardy [3] for the special case $n=2$.)

From the periodic nature of $B_{0}$ it is trivial that when $\eta \equiv 0(\bmod A)$, $B_{0}=B_{0}\left(\eta ; a_{1}, \cdots, a_{n}\right)=B_{0}\left(0 ; a_{1}, \cdots, a_{n}\right)$. The $B_{i}$ are found as follows:

Our function $f(S)$ has:

(i) A pole of order $n+1$ at $S=0$. The residue $P(\eta)$ here is a polynomial in $\eta$ with coefficients which, when multiplied by $A$, are polynomials (with rational coefficients) in the $a_{i}$. This remark is true for all non-negative real $\eta$.

(ii) A pole of order $n$ at $S=2 m \pi i$ ( $m$ an integer $\neq 0)$. Here again, the residue $Q(\eta)$ is a polynomial in $\eta$ with coefficients which, when multiplied by $A$, are polynomials (with rational coefficients) of the $a_{i}$ provided $\eta$ is an integer.

(iii) Simple poles at $S=2 g_{i} \pi i / a_{i}$ where $g_{i} \not \equiv 0\left(\bmod a_{i}\right)$. The residue here is denoted by $T_{i}(\eta)$, and has a period $a_{i}$. (The argument in Hardy [3] generalizes at once to prove this statement.) $T_{i}(\eta)$ is an infinite trigonometrical series. (Incidentally, in another note we show how to reduce these to finite trigonometrical sums.) Thus ${ }^{3}$

\footnotetext{
s In fact for all $\eta \geqq 0, T_{i}(\eta)=\sum_{0_{i} \not 0\left(\bmod a_{i}\right)} \exp \left(\eta\left(2 g_{i} \pi i / a_{i}\right)\right) / 2 g_{i} \pi i \cdot H_{i}$, where $H_{i}$ $=\prod_{u \neq i}\left(1-\exp \left(\left(-2 g_{i} \pi i a_{u} / a_{i}\right)\right)\right.$.
} 


$$
L^{*}(\eta)=P(\eta)+Q(\eta)+\sum_{i=1}^{n} T_{i}(\eta)[\eta \text { an integer } \geqq 0] .
$$

Finally we evaluate $r(\eta)$ when $\eta \equiv 0(\bmod A)$ as follows. The latter condition on $\eta$ holds throughout the rest of the paper, and we shall not repeat it. Similar to our proof of (7) we easily establish that

(8) $\quad L^{*}(\eta+1 / 2)=P(\eta+1 / 2)+R(\eta+1 / 2)+\sum_{i=1}^{n} T_{i}(\eta+1 / 2)$.

The $R$ function here is not the same as the $Q$ introduced before. However, it remains, like $Q$, a polynomial in $\eta$ with coefficients which, when multiplied by $A$, are polynomials (with rational coefficients) in the $a_{i}$. Observe that

$$
\begin{aligned}
\sum_{i=1}^{n} T_{i}\left(\eta+\frac{1}{2}\right) & =\sum_{i=1}^{n} T_{i}\left(\frac{1}{2}\right) \\
& =L^{*}\left(\frac{1}{2}\right)-P\left(\frac{1}{2}\right)-R\left(\frac{1}{2}\right) \\
& =1-P\left(\frac{1}{2}\right)-R\left(\frac{1}{2}\right)
\end{aligned}
$$

and

$$
r(\eta)=2\left\{L^{*}(\eta+1 / 2)-L^{*}(\eta)\right\} .
$$

From the above equations we see that Theorems 1 and 2 follow. In fact $r(\eta)$ is a polynomial in $\eta$ of degree $n-1$ with coefficients which, when multiplied by $A$, are polynomials (with rational coefficients) in the $a_{i}$. An identical remark is true about $L(\eta)$, except that we have, in this case, a polynomial of degree $n$ in $\eta$.

\section{REFERENCES}

1. D. H. Lehmer, The lattice points of an n-dimensional tetrahedron, Duke Math. J. vol. 7 (1940) pp. 341-353.

2. D. C. Spencer, The lattice points of tetrahedra, Journal of Mathematics and Physics vol. 21 (1942) pp. 189-197.

3. G. H. Hardy, Ramanujan, Cambridge University Press, 1940.

UNIVERSITY OF CoLORADO AND UNIVERSITY OF MASSACHUSETTS 